\title{
Rheumapatienten in der Pandemie
}

Die SARS-CoV-2-Pandemie betrifft Patienten mit auto-inflammatorischen entzündlichen Erkrankungen in besonderem Maße.

Coronaviren sind uns lange bekannt und für etwa 10 bis $15 \%$ aller milden, meist saisonalen Infekte des oberen Respirationstraktes verantwortlich. Im Dezember 2019 trat erstmals das „neue" Coronavirus SARS-CoV-2 als Erreger der pandemisch gewordenen COVID-19 Erkrankung auf. Die Herkunft des SARS-CoV-2 ist nach wie vor nicht vollständig geklärt. Es wird angenommen, dass das Ursprungstier dieser zoonotischen Infektion die Fledermaus ist, wobei ein oder mehrere Zwischenwirte wie etwa das Schuppentier wahrscheinlich ebenso an der Übertragung auf den Menschen beteiligt sind. Über den zellulären Angiotensin Converting Enzyme (ACE)-2-Rezeptor, der in der Nasenschleimhaut, an den Alveolarepithelzellen, sowie in vielen anderen Organen vorhanden ist, gelangt das Virus mit Hilfe seines "Spike“-Proteins und einer zellulären Serinprotease in die Wirtszelle. Das Krankheitsbild von COVID-19 ist mittlerweile gut untersucht und betrifft in erster Linie den Respirationstrakt, aber auch alle anderen Organsysteme können mit betroffen sein. Nach einer medianen Inkubationszeit von fünf Tagen zeigen sich die klassischen Symptome einer Virusinfektion mit erhöhten Körpertemperaturen, Glieder- und Muskelschmerzen, Kopfschmerzen, Müdigkeit sowie ein trockener Husten. Bei etwa $10 \%$ der Erkrankten treten auch gastrointestinale Symptome wie Durchfall und Übelkeit auf. Suggestive Symptome für COVID-19 sind auch Störungen des Geschmacks- und Geruchssinns bis hin zur Anosmie und Ageusie. Das Spektrum der Erkrankung reicht von asymptomatischen/wenig symptomatischen bzw. relativ milden Verläufen, bis hin zur Notwendigkeit der Hospitalisierung und der Intensivver- sorgung. Typischerweise dauert es ca. 7 Tage, bis nach Erkrankungsbeginn eine Hospitalisierung meistens aufgrund zunehmender respiratorischer Beschwerden wie Dyspnoe erforderlich ist. Als Faustregel gilt, dass etwa $20 \%$ aller Erkrankten einen Spitalsaufenthalt und davon $1 \%$ eine intensivmedizinische Betreuung benötigen. Das Risiko für schwere Verläufe ist abhängig von Risikofaktoren wie fortgeschrittenes Alter, schlecht eingestelltes metabolisches Syndrom mit Diabetes, Adipositas, schlecht kontrollierter Bluthochdruck, schwere kardiale Vorerkrankungen oder fortgeschrittene chronische Lungenerkrankungen. Männliches Geschlecht ist ebenso häufiger mit der Notwendigkeit der Hospitalisierung und auch schwereren Verläufen assoziiert. Inwieweit die Blutgruppe A mit einer höheren InfektionsAnfälligkeit verbunden ist, ist noch in reger Diskussion. Relativ typisch, aber nicht spezifisch für COVID-19 sind die fleckförmigen milchglasartigen Veränderungen in der Thorax-Computertomographie, die in Kombination mit der entsprechenden Klinik und der epidemischen Situation auf eine Infektion mit dem SARS-CoV-2 hinweisen können. Patientinnen und Patienten mit autoimmunen/auto-inflammatorischen entzündlichen Erkrankungen waren und sind in besonderem Maße von der SARS-CoV-2 Pandemie betroffen.

\section{》) Glukokortikoide sollten in}

möglichst niedriger Dosis verabreicht werden

\section{Erhöhtes Risiko nicht bestätigt}

Es ist nach Verordnung des Bundesministeriums für Soziales, Gesundheit,
Pflege und Konsumentenschutz für diese Patienten eine individuelle Einschätzung hinsichtlich eines schweren Verlaufes (=Risikopatienten) vorzunehmen und sie sind entsprechend zu schützen. Das impliziert eine durch die Grundkrankheit bzw. durch die immunsuppressive/immunmodulatorische Therapie angenommene erhöhte Anfälligkeit für eine SARSCoV2 Infektion und/oder einen schwereren Verlauf. Diese Annahme konnte bis dato im Wesentlichen nicht bestätigt werden, da für Patienten mit chronisch entzündlichen rheumatischen Erkrankungen und ihren Therapien kein erhöhtes COVID-19 Risiko gezeigt wurde. Eine Ausnahme davon betrifft die Glukokortikoidtherapie, die nach den vorliegenden Daten sowohl die Anfälligkeit für die SARS-CoV-2 Infektion wie auch das Risiko für schwerere Verläufe der COVID-19 Erkrankung erhöht. Dies lässt sich aus den Daten internationaler Register für rheumatische und auch gastroenterologische (IBD) Erkrankungen ersehen. Glukokortikoide sollten demnach in möglichst niedriger Dosis verabreicht werden. Für bestehenden Biologika oder andere Basistherapien besteht allerdings keinerlei Grund, diese präventiv zu pausieren bzw. entsprechend notwendige Therapien NICHT einzuleiten. Dementsprechende Empfehlungen wurden von allen Fachgesellschaften (ÖGR, EULAR, ÖGGH, ÖGDV, AAD) mehrfach publiziert.

\section{Hydroxychloroquin}

In der Rheumatologie vielfach eingesetzte Substanzen wurden als Therapien der COVID-19 Erkrankung diskutiert und bereits breit eingesetzt, obwohl deren Wirksamkeit bei der Virusinfektion noch lange nicht eta-

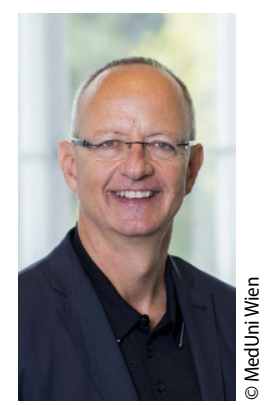

Prof. Dr. Stefan Winkler, MedUni Wien 
Erstveröffentlichung im skriptum Kongressjournal 9/2020: 17-18 bliert war. So kam es durchaus zu Engpässen bei der Verfügbarkeit von z. B. Hydroxychloroquin für Rheuma- $\mathrm{Pa}-$ tienten. Die mittlerweile nachgewiesene Unwirksamkeit von Hydroxychloroquin sowohl bei der Prävention als auch in der Therapie von COVID-19 beendete diesen Engpass allerdings sehr rasch.

\section{Therapie der Hyperinflammation}

„Rheuma"-Therapien waren und sind nach wie vor in der Therapie der so genannten Hyperinflammation der schweren COVID- 19 Erkrankung in Diskussion und auch in einigen Studien untersucht. Diese Hyperinflammation, auch „Zytokinsturm" (Cytokine Release Syndrome) genannt, stellt eine Grundlage für unkontrollierte und lebensbedrohliche COVID-19 Verläufe dar. Obwohl vielleicht dieser Zytokinsturm in der Pathogenese doch etwas überschätzt wurde wie neuere Publikationen nahelegen, wird sehr viel Augenmerk auf eine Behandlung desselben gelegt. Eine lange Liste an Substanzen - viele davon in der Rheumatologie wohlbekannt - kommt für die anti-inflammatorische Therapie bei schweren Covid-19 Erkrankungen ins Spiel:

- IL-6-Inhibitoren (Tocilizumab, Clazakizumab)

- JAK-Inhibition (z. B. Baricitinib)

- TNF-a-Inhibitoren

- IL-1-Blockade (Anakinra, Colchizin, Canakinumab)

- IVIG

- Cortison

- Abatacept

- Interferone (auch IFN- $\lambda$ )

- IL-7

- IL-33-Inhibition

- BCG-Impfung

- Tyrosinkinase-Hemmer

- usw.

Ein klares Therapiekonzept lässt sich aus diesen Behandlungsvorschlägen zurzeit noch nicht ablesen. Insbesondere liegen keine Studienergebnisse vor, die einen Rückschluss auf eine gezielte Behandlung von COVID-19 bzw. auch auf eine Behandlung der Komplikationen erlauben würden. Lediglich für Dexamethason konnte in der RECOVERY (Randomised Evaluation Of COVID-19 Therapy) Studie der Universität Oxford gezeigt werden, dass die Mortalität von beatmeten Erkrankten um ein Drittel gesenkt werden konnte. Für einen etwaigen Erfolg der Glukokortikoide spielt wohl der Zeitpunkt des Einsatzes der Therapie im Laufe der Erkrankung eine entscheidende Rolle. Leider bestehen bis dato keinerlei andere Therapieoptionen bei der pandemischen Coronavirus-Erkrankung, sodass dringend auf eine wirksame Impfung gehofft werden muss. Die Frage, inwieweit Impfstoffe eine anhaltende Immunität gerade bei unseren immunmodulierten/immunsupprimierten Rheuma-Patienten induzieren können, kann momentan allerdings nicht beantwortet werden. (Stand der Information 11.2020)

\section{Prof. Dr. Stefan Winkler}

Hinweis des Verlags. Der Verlag bleibt in Hinblick auf geografische Zuordnungen und Gebietsbezeichnungen in veröffentlichten Karten und Institutsadressen neutral.

rheuma plus $2021 \cdot 20: 19-20$ https://doi.org/10.1007/s12688021-00409-y

(C) Springer-Verlag GmbH Austria, ein Teil von Springer Nature 2021

Hier steht eine Anzeige. 